Saberes clásicos

y nuevos saberes 



\title{
Diálogo de saberes entre la cultura escolar y la cultura digital en el Nivel Medio: oportunidades y desafíos
}

Rita De Pascuale

Universidad Nacional del Comahue, Argentina

ritapascuale@gmail.com

\author{
Sonia Sansot \\ Universidad Nacional del Comahue, Argentina \\ soniasansot@gmail.com
}

\begin{abstract}
Resumen
El presente artículo se deriva de un proyecto de investigación centrado en las Tecnologías de la Información y la Comunicación (TIC) que median las prácticas de enseñanza en el Nivel Medio en las provincias de Neuquén y Río Negro. Pretende describir y comprender la complejidad de las prácticas de enseñanza considerando las TIC como instrumento de mediación. La investigación es de carácter exploratorio y se inscribe en una perspectiva metodológica cualitativa. Desde el marco teórico-metodológico de la tercera generación de la Teoría de la Actividad, se analizan prácticas de enseñanza mediadas por TIC, identificando Sistemas de Actividad entramados en una red: uno referido al sujeto de enseñanza, otro al sujeto de aprendizaje, y la emergencia de un tercero sobreCompetencias Digitales. Sus interacciones y contradicciones permitencomprender el diálogo de saberes entre lacultura digital y la cultura escolar.La cultura digitalmodifica los contextos de aprendizaje cotidianos e interpela a la cultura escolaren sus modos de transmisión y construcción de saberes, formación y constitución de subjetividades. Este diálogo requiere comunidades de práctica que promuevan la formulación y reflexión sobre nuevos repertorios de actuación para hacer usos más complejos, significativos,creativos y seguros de las tecnologías digitales.
\end{abstract}

Palabras clave: Enseñanza mediada por TIC, Competencia Digital, Teoría de la Actividad, Sistema de Actividad 


\title{
Dialogue of knowledge between school culture and digital culture in Secondary School: opportunities and challenges
}

\begin{abstract}
This article stems from an investigation project focused on Information and Communication Technologies in secondary school teaching practices in the provinces of Neuquén and Rio Negro. It attempted to describe and understand the complexity of teaching practices taking into account ICT as a mediation instrument. The investigation is exploratory and its methodology is qualitative. The teaching practices mediated by ICT were analysed within the theoretical-methodological framework of the third generation of the Activity Theory. Different Activity Systems, which were integrated in a net, were identified: one of them refers to the teaching subject, another to the learning subject and a third one to Digital Competences. Their interactions and contradictions enabled to understand the dialogue of knowledge between the Digital Culture and the School culture. The Digital Culture modifies daily learning contexts and questions the School Culture about knowledge construction and transmission, training and constitution of subjectivities. This dialogue requires teaching practices that promote the formulation of and reflection on new ways to use digital technologies in a more complex, creative, significant and safe manner.
\end{abstract}

Keywords: Teaching mediated by ICT, Digital Competence, Activity Theory, Activity System

\section{Introducción}

Esteartículose deriva del proyecto de investigación Las prácticas de enseñanza mediadas por TICdesarrollado entre 2014 y 2017 en la Facultad de Ciencias de la Educación de la Universidad Nacional del Comahue. El estudio se centró en las Tecnologías de la Información y la Comunicación (TIC) que median las prácticas de enseñanza en el Nivel Medio. Las TIC se entienden como un instrumento de mediación que aparece como novedad -en la última década- en el proceso de enseñar y aprender. La investigación se sostiene en un marco teórico en el cual convergen la Psicología-desde la perspectiva de la Cognición Situada- y la Didáctica -desde la perspectiva reconceptualista- para describir y comprender la complejidad de las prácticas de enseñanza en el Nivel Medio, a partir del estudio de un instrumento de mediación específico: las TIC.

Se conceptualizalas prácticas de enseñanza como una actividad definida públicamente cuya intencionalidad es promover procesos de aprendizaje en los/as estudiantes, y los instrumentos de mediación como herramientas (físicas o psicológicas) que tienen un origen social y son utilizadas para comunicarse con otros, mediar en el contacto de los mundos sociales y luego internalizar su uso. Ambos conceptos asignan un papel esencial a la escolarización en tanto crea contextos sociales para dominar y ser conscientes del uso de herramientas culturales.

La Escuela Media se considera aquí como el escenario donde se despliegan las prácticas de enseñanza, conceptualizando este escenario como un espacio temporal organizado física, económica, política y socialmente para desarrollar una actividad. Dicho escenario no es directamente negociable por el individuo, es exterior a él y no obstante lo abarca, proporcionando un marco institucional de orden superior en el que se constituye el ambiente. Este escenario está inmerso hoy en tensiones y contradicciones que generan un cambio histórico que desinstituye y modifica el entramado construido por 
la modernidad. Las culturas han cambiado profundamente, lo multimodal permea y se incorpora a la vida escolar, sin reconocer que -en la mayoría de los casos- ambos escenarios (medios-escuelas) portan lógicas diferentes y ofrecen respuestas diferentes a las preguntas por el enseñar y el aprender.

Laescuela media se debate entre los dispositivos construidos en la modernidad y los nuevos dispositivos de esta modernidad líquida (Bauman, 2013). La persistencia de los viejos dispositivos esuna fuente inagotable de contradicciones y conflictos al interior del nuevo escenario escolar. El desarrollo de prácticas letradas constituyó tradicionalmente uno de los objetivos centrales de la escolarización, sin embargo en la actualidad las TIC amplían y complejizan el escenario con nuevos saberes cuyo dominio es necesario para desenvolverse en el marco de sociedades mediatizadas.

El escenario de los medios muestra un mundo fragmentado y veloz que formatea rasgos cognitivos novedosos: "pensamiento atomizado, sin jerarquización semántica, no relacional, no explicativo, no argumentativo"(Litwin, 2008: 144), rasgos totalmente opuestos a los que intenta desarrollar la escuela. Sin embargo, la familiaridad con estos medios hace que se vuelvan invisibles, es decir que no somos conscientes de los cambios que provoca su utilización y no permite identificar cómo condicionan las prácticas del enseñar y del aprender. En efecto, nuevas culturas comunicacionales aportan nuevos conocimientos (técnicos y culturales) que se materializan en los medios y las formas comunicativas derivadas de una Cultura Digital que modela nuestras prácticas sociales.

Las transformaciones en la producción de significados (por tecnologías y medios) modifican los contextos de aprendizaje cotidianos caracterizados por experiencias vivenciales y de fascinación que interpelan la cultura escolaren sus modos de transmisión de saberes, formación y constitución de subjetividades. En este marco, interesa indagar: ¿ cómo es el escenario de la escuela media atravesado por TIC? ¿Qué características asumen las TIC como instrumento de mediación en las prácticas de enseñar? ¿Cómo median en la construcción de las propuestas de enseñanza? Y Y cuál es el sentido que docentes y estudiantes le otorgan a las TIC en la construcción del conocimiento?

Afrontar las posibilidades y desafíos de las tecnologías digitales es cada vez más necesario para promover su apropiación y contribuir a disminuir desigualdades;propiciar en nuestros/as estudiantes el pasaje de receptores a creadores ya no es solo un asunto didáctico y ético sino profundamente político.Específicamente en relación con su praxis comunicacional,MartínBarbero (2001) asigna importancia estratégica auna escuela capaz de favorecer un uso creativo y crítico de los mediosaudiovisuales y las tecnologías digitales como espacios y herramientas claves. Es necesario que el sistemaeducativo transite de un modelo centrado en la secuencia linealque encadena "unidireccionalmente" grados, edades y paquetes de conocimiento a otro "descentrado y plural"; además es necesario integrar a la formación el uso seguro en las redes. Para ello, consideramos pertinente el planteo que sobre Competencias Digitales realiza el Parlamento Europeo:

\footnotetext{
... implica el uso crítico y seguro de las Tecnologías de la Sociedad de la Información para el trabajo, el tiempo libre y la comunicación. Apoyándose en habilidades TIC básicas: uso de ordenadores para recuperar, evaluar, almacenar, producir, presentar e intercambiar información y para comunicar y participar en redes de colaboración a través de Internet. (2006, en INTEF, 2017)
}

Los aportes de la tercera generación de la Teoría de la Actividad son cruciales para el abordaje teórico-metodológico. Resultan potentes para entender el diálogo de saberes entre culturas, las múltiples perspectivas y las redes de interacción de los sistemas de actividad que aquí se analizan a partir de sus cinco principios (Engeström, 2001a): el sistema de actividad como unidad de análisis, la multiplicidad de voces en la actividad, 
la historicidad de la actividad, las contradicciones como motor de cambio y los ciclos expansivos como posible forma de transformación de la actividad.

Analizamos las prácticas de la enseñanza mediadas por TIC identificando Sistemas de Actividad entramados en una RED: uno referido al sujeto de enseñanza, otro al sujeto de aprendizajey un tercero derivado de ellos. El primer Sistema de Actividad permitió reconocer la necesidad de que la Institución educativa conforme una comunidad de práctica que acompañe al docente en la integración de TIC. En el segundo Sistema de Actividad,los/las estudiantesportan saberes como usuarios intensivos de TIC en su vida cotidiana, creando expectativas sobre su competencia digital.

La emergencia del tercer Sistema de Actividad está dada por la contradicción primaria que supone el ingreso de nuevas subjetividades conformadas en la convergencia digital (Canclini, 2007) que tensionan las prácticas sedimentadas de la cultura escolar.El encuentro de estudiantes con saberes tecnológicos instrumentalesy docentes que portan saberes consolidados desde una cultura letrada invierte roles históricos y jaqueaala autoridad pedagógica.Al modificarse un componente, todo el Sistema adquiere cualidades diferentes, en este caso, el "uso pedagógico" de las tecnologías plantea contradicciones internas y de las maneras en que se resuelvan dependerá su desarrollo.

\section{Aspectos teórico-metodológicos}

Las propuestas de la Teoría de la Actividad, si bien focalizan en la cognición distribuida en contextos de participación social, en su conjunto son sugerentes para analizar las prácticas de enseñanza y aprendizaje en el marco de un sistema educativo fuerte y constantemente interpelado por exigencias de adecuación a emergentes demandas sociales. Los estudios sobre el desarrollo de la cognición constituyen una de las tendencias más representativas de la teoría y la actividad sociocultural (Daniels, 2003). Recientemente se han incrementado los enfoques que investigan el desarrollo de la cognición en su contexto, entre los cuales, según Daniels (2003) se encuentran: teoría de la actividad cultural-histórica (Cole, 1999); enfoques socioculturales (Wertsch, 1993); modelos de aprendizaje situado (Lave, 1991 y Wenger, 2001) y enfoques basados en la cognición distribuida (Salomon, 1993 y Engeström, 2001b).

La emergencia de enfoques que investigan el desarrollo de la cognición en su contexto se opone a prácticas escolares que asumen que el conocimiento puede abstraerse de las situaciones en que se aprende y emplea. Se destaca la importancia de la actividad y el contexto en los procesos del enseñar y del aprender interpelando la manera en que la institución escolar intenta promover el aprendizaje. Cuestionan la forma en que se enseñan conocimientos declarativos, abstractos y descontextualizados, inertes y de relevancia social limitada (Díaz Barriga, Frida, 2003). Conciben el aprendizaje distribuido en contextos de participación y no exclusivamente en la cabeza de las personas, enfatizando el involucramiento del aprendiz que participa comprometidamente en prácticas con otros.

Para estos estudios, resulta central la categoría de actividad originada en los desarrollos de Vigotsky al postular que la acción humana está mediada por herramientas (físicas o simbólicas). Esta categoría es la unidad de análisis básica de la acción cultural humana y consiste en la acción de un sujeto sobre la realidad objeto mediante herramientas. Estos elementos integran una unidad, la comprensión de las formas de pensamiento están imbricadas en las prácticas. La actividad no se realiza directamente sobre la realidad sino a través de la mediación de un instrumento. Esta concepción de mediación integra de tal manera la herramienta a una práctica determinada que, sin ella, esa 
práctica no se podría comprender. $\mathrm{Al}$ actuar sobre la realidad usando herramientas, estas forman parte de la cognición que orienta la acción. La actividad tampoco se realiza en soledad, sino en el marco de un Sistema de Actividad que integra sujeto, objeto e instrumentos y se lleva a cabo en una comunidad, regulada por un conjunto de reglas y una determinada división social del trabajo. Ello define una comunidad de prácticas que comparte cierta identidad en las prácticas cotidianas.

En línea con Baquero y Terigi (1996), se plantea aquí una situación de aprendizaje concebida como sistema de actividad: un sujeto que aprende, instrumentos utilizados en la actividad(privilegiadamente semióticos), un objeto $u$ objetivo a apropiarse que regula la actividad (saberes y contenidos), una comunidad de referencia en que actividad y sujeto se insertan, reglas que regulan las relaciones sociales y una división del trabajo. Cada componente del sistema se define por su posición subjetiva, relativa y recíproca en dispositivos institucionales que se articulan indefinida y dinámicamente. Consideramos que la Teoría de la Actividad aporta una unidad de análisis que responde a la necesidad, señalada por estos autores, de ampliar la mirada cognitiva más allá de la relación sujeto-objeto, situando esta relación en el contexto de una actividad cultural específica. Los instrumentos de mediación -en este caso las TIC- no son herramientas neutras a través de las cuales se ejecutan operaciones; integran las comprensiones de las prácticas en que se usan. Esto es particularmente importante ya que moldearán modalidades de apropiación y uso de instrumentos semióticos en la escolarización.

Nuestra investigación se inscribió en una perspectiva metodológica cualitativa de carácter exploratorio. El trabajo de campo se realizó en los últimos años de Instituciones de Nivel Medio en lasprovincias de Neuquén y Río Negro, con la participación dedocentes de seis instituciones, a saber:

\begin{tabular}{lll}
\hline \multirow{2}{*}{ Neuquén } & \multicolumn{1}{c}{ Cipolletti } & \multicolumn{1}{c}{ Río Negro } \\
\cline { 2 - 3 } & \multicolumn{2}{c}{ Ing. Jacobacci } \\
\hline Esc. Pcial de Educación & 2 Centros de Educación & 1 Centro de Educación Técnica \\
\cline { 3 - 3 } $\begin{array}{l}\text { Media } \\
\text { Educación Media }\end{array}$ & & 1 Centro de Educ. Media \\
\hline
\end{tabular}

Inicialmente se realizó una aproximación exploratoria a las prácticas de enseñanza mediadas por TIC mediante una encuesta autoadministrada (online) para identificar a informantes clave.Se utilizó una aplicación web gratuita que permite sondear un grupo relativamente numeroso en un tiempo mínimo y con un dispositivo digital cotidiano (celular).Se definieron tres dimensiones con sus indicadores:

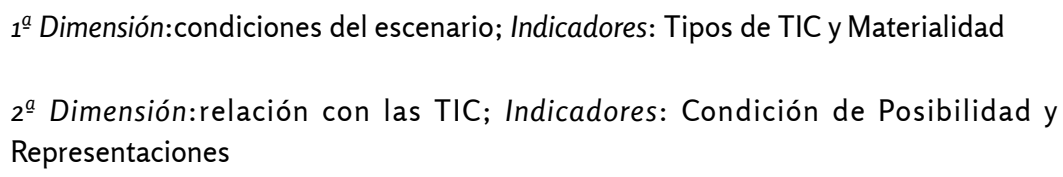

Para su autoadministración, la encuesta se habilitó en un BLOG especialmente construido. Se trató de un sitio público que posibilitó acceso tanto al proyecto como a las distintas producciones que sobre el tema produjera el equipo. No obstante ello, pasado elplazo establecido, la participación fue muy escasa (6 docentes) por lo cual se decidió imprimir la encuesta y distribuirla en las instituciones seleccionadas inicialmente, obteniendo los siguientes resultados: 


\begin{tabular}{|c|c|c|c|c|}
\hline \multirow{2}{*}{ Ciudad } & \multirow{2}{*}{\multicolumn{2}{|c|}{$\begin{array}{l}\text { Institución } \\
\text { Online }\end{array}$}} & \multicolumn{2}{|c|}{ Encuesta } \\
\hline & & & Impresa & \\
\hline \multirow{2}{*}{ Neuquén } & 1 & EPET & 5 & 4 \\
\hline & 2 & CEPM & 1 & 1 \\
\hline \multirow{2}{*}{ Cipolletti } & 3 & CEM & o & 2 \\
\hline & 4 & CEM & o & 10 \\
\hline \multirow{4}{*}{ Ing. Jacobacci } & 5 & CET & o & 5 \\
\hline & 6 & CEM & 0 & 13 \\
\hline & & Subtotal: & 6 & 35 \\
\hline & & Total: & \multicolumn{2}{|c|}{41} \\
\hline
\end{tabular}

Esta etapa permitió realizar un primer nivel de análisis y ajustar la construcción del siguiente instrumento metodológico previsto: la entrevista en profundidad a informantes clave. El protocolo consideró las mismas dimensiones enunciadas. Se administraron intencionalmente a 11 docentes que en la encuesta -a nuestro juicio- dieron cuenta de un trabajo didáctico con las TIC.

\section{Contradicciones del Sistema de Actividad}

Con base en los marcos referenciales precedentes, esta investigación sostiene que estudiar las prácticas de la enseñanza mediadas por TIC, en el escenario del Nivel Medioinmerso en sociedades mediatizadas, resulta relevante para reconocer los distintos ambientes que las instituciones construyen en él. El escenario de la escuela media analizadoen las provincias de Río Negro y Neuquén se encuentra en un proceso de transición en donde, por ahora, pareciera solo reconocerse la necesidad de incorporar las TIC a la prácticas de enseñar y aprender más vinculadas a la cultura escolar de la modernidad,lo cual obstaculizaríasu uso didáctico. Bajo este supuesto, nos preguntamos si es posible construir conocimiento mediado por TIC que configure herramientas válidas de intervención docente para promover el diálogo de saberesentre la cultura escolar y la cultura digital en el Nivel Medio.

Cabe destacar que al momento de realizar este estudio (2014-2017) se estaba reformulando el Currículum de Nivel Medio en la provincia de Río Negro. Entre los avances que se plantearon, se consideró la posibilidad de extender la jornada y realizar una revisión en profundidad de los contenidos que se sostenían curricularmente. Esta propuesta de reforma curricular por parte de la Secretaría de Educación de la Provincia fue cuestionada por el Gremio Docente dado que no contaba con el acompañamiento presupuestario correspondiente. Por su parte, en Neuquén coexistían diversas Currículas, más vinculadas a proyectos educativos institucionales que a uno Oficial. En ambas provincias, el Programa Conectar Igualdad tuvo amplio consenso y distribución de equipamiento para la disminuir la brecha digital. Se trató de promover el acceso a la tecnología pero sin repercusiones a nivel de la discusión curricular que se estaba desarrollando(desconsiderando las condiciones institucionales), ni a nivel de las prácticas de enseñanza ni las de aprendizaje, tal como puede apreciarse a continuación:

D:-La tecnología que más trascendencia ha tenido es lo vinculado con Conectar Igualdad, no obstante eso, antes se utilizaba también televisor, grabador, retroproyector, cañón; con la incorporación de las computadoras a los alumnos es toda una cuestión, tiene sus ventajas y sus desventajas. La ventaja es la posibilidad de compartir información de manera más ágil, más rápida que con las fotocopias, pero eso no garantiza que lo lean o que lo aprendan. Acá en el colegio funciona un servidor, los 
profesores tienen una carpeta, suben el contenido que quieran, ya sea teoría, videos, fotos y los alumnos lo pueden bajar, es la posibilidad que hay en el colegio,no hay conexión a Internet en las aulas. Eso sería lo formal.(Entrevista 4:13)

Para comprender la complejidad del escenario en que se desarrollan las prácticas de enseñanza mediadas por TIC, resulta fructífero el Modelo de los Saberes Tecnológicos y Pedagógicos del Contenido (TPACK por sus siglas en Inglés) propuesto por Koheler, Mishra y Cain (2015). El modelo TPACK refiere a la comprensión de la interacción entre tres saberes docentes. Permite reconocer la experticia del docente sobre el Contenido de la disciplina que enseña; mientras que la experticia sobre la enseñanza refiere al Contenido Pedagógico, entendido como el conocimiento profundo de los/as docentes sobre los procesos de enseñanza y aprendizaje. Los Conocimientos sobre Tecnología resultan difíciles de formalizar dado su fluidez permanente y carácter provisorio, pero permiten desarrollar una variedad de tareas usando tecnología.

El Modelo TPACK es más que la suma de sus componentes, hace referenciaa la interacción entre los tres saberes mencionados. Requiere una comprensión de la representación de conceptos, usando habilidades tecnológicas y pedagógicas de manera constructiva. Estas prácticas de enseñanza situadas están condicionadas por la habilidad docente para desenvolverse con flexibilidad en el ambiente de la clase y reconocer la trama de interacciones entre estos saberes.Sibien los Sistemas de Actividad analizados se orientan a la integración de TIC, también reconocen al sujeto de aprendizaje como portador de prácticas sociales emergentes que resultan del desarrollo de algunas de las dimensiones de la Competencia Digital(CD) ligadas, fundamentalmente a la comunicación y al acceso a información.

En su conceptualización más amplia, la competencia digital implica "el uso crítico y seguro de las Tecnologías de la Sociedad de la Información para el trabajo, el tiempo libre y la comunicación"(EuropeanParliament and the Council, 2006, en INTEF, 2017). Estas indicaciones implican las siguientes cinco áreas que conforman la CD:

1. Información y alfabetización informacional: identificar, localizar, recuperar, almacenar, organizar y analizar la información digital, evaluando su finalidad y relevancia.

2. Comunicación y colaboración: comunicar en entornos digitales, compartir recursos a través de herramientas en línea, conectar y colaborar con otros a través de herramientas digitales, interactuar y participar en comunidades y redes; conciencia intercultural.

3. Creación de contenido digital: crear y editar contenidos nuevos (textos, imágenes, videos), integrar y reelaborar conocimientos y contenidos previos, realizar producciones artísticas, contenidos multimedia y programación informática, aplicar los derechos de propiedad intelectual y las licencias de uso.

4. Seguridad: protección personal, protección de datos, protección de la identidad digital, uso de seguridad, uso seguro y sostenible.

5. Resolución de problemas: identificar necesidades y recursos digitales, tomar decisiones a la hora de elegir la herramienta digital apropiada, acorde a la finalidad o necesidad, resolver problemas conceptuales a través de medios digitales, resolver problemas técnicos, uso creativo de la tecnología, actualizar la competencia propia y la de otros.

$\mathrm{Al}$ considerar estas cinco dimensiones en su conjunto, posiblemente pensar y diseñar propuestas para desarrollar CD adquiera otra complejidad. Estas áreas suponen desplegar en la enseñanza procesos de co-creación e interpelaciones a las prácticas tradicionales y a las relaciones verticales que impliquen a las nuevas subjetividadesy 
potencien su libertad creativa y estética de manera segura. Desde esta perspectiva, la problematización de prácticas escolares cristalizadas requiere reflexión sobre las acciones y decisiones prácticas en diálogo con la innovación propuesta, la confrontación con otras experiencias similares y, fundamentalmente, la escucha y consideración de las demandas y posibilidades de los/las estudiantes.Cabe recordar que, al modificarse un componente, el Sistema en su totalidad adquiere cualidades diferentes, en este caso, el "uso pedagógico" de las tecnologías plantea contradicciones internas (flechas quebradas en el siguiente gráfico).

En relación con las dimensiones y categorías construidas en este estudio, analizamos las prácticas de la enseñanza mediadas por TIC identificando sus sistemas de actividad entramados en una RED de Sistemas: uno referido al sujeto de enseñanza que integra TIC, otro referido al sujeto de aprendizaje- usuario de redes sociales, y otro referido a las competencias digitales, tal como puede verse en la siguiente figura:

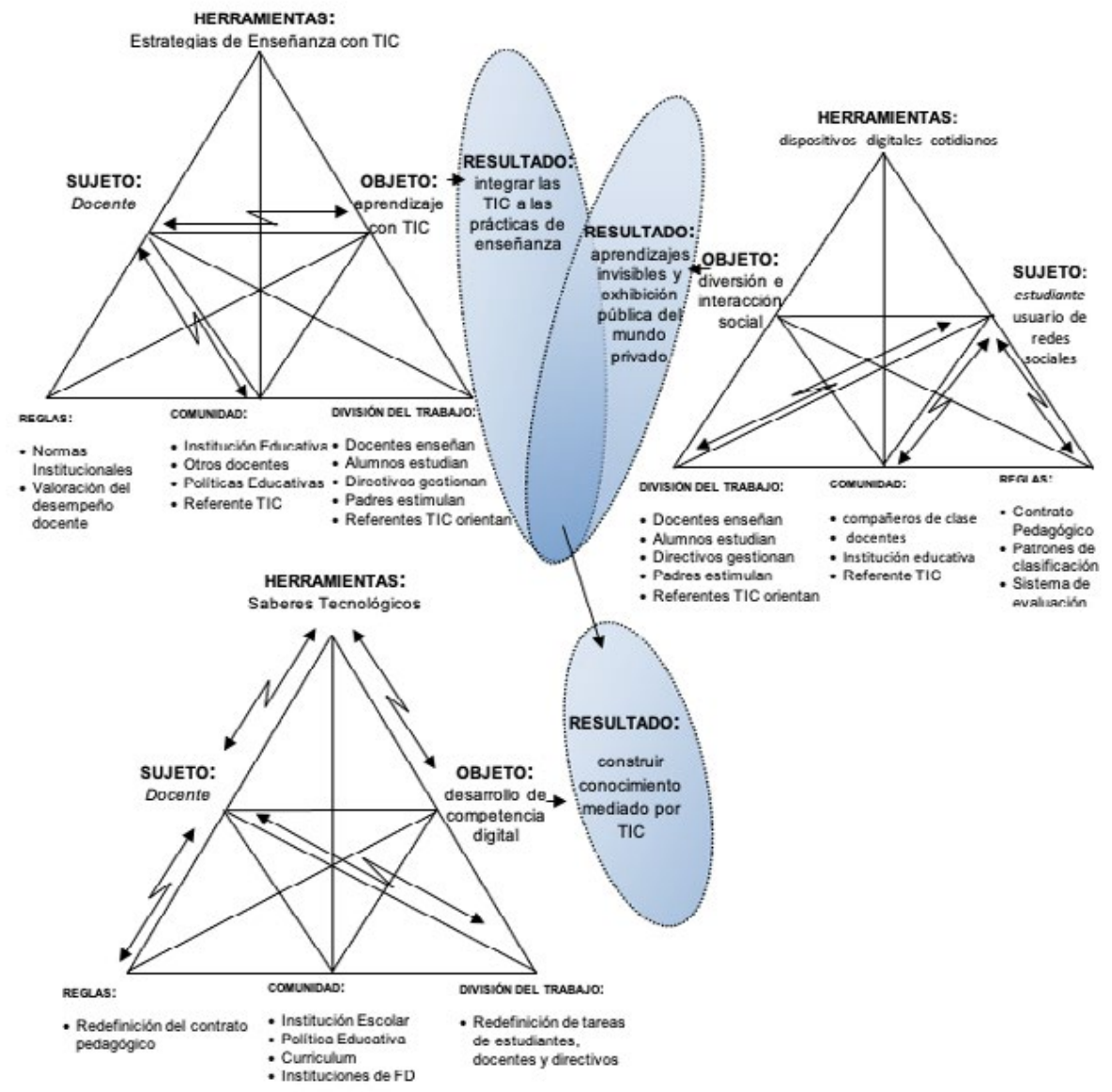

El requerimientode entramar Saberes Tecnológicos en las prácticas de enseñanza genera contradicciones al interior del Sistema dado que al docente hasta el momento se le demanda una doble experticia: en la disciplina y en su enseñanza. Particularmente la clase con TIC, concebida como un sistema de actividad, porta condiciones novedosas relacionadas con los Saberes Tecnológicos como herramienta de mediación.

Esto se vincula a un modo de pensar el conocimiento y su circulación en la clase y, por ende, influye en los modos de apropiación. Tal como plantea Edwards, "la presentación 
del conocimiento en formas distintas le da significaciones distintas y lo altera como tal, además tiene consecuencias para el grado de apropiación posible del conocimiento por los sujetos" (1985: 7).Si la forma es contenido, se supone que el entramado de saberes tecnológicos y conocimiento permitiría al sujeto de aprendizajeentablar una relación de interioridad con el saber, es decir, una relación significativa cuando el conocimiento que se presenta incluye e interroga al sujeto. Sin embargo, en el primer Sistema de Actividad identificamos que al intentar este entramado,lasprácticas pueden ser interpeladas y resistidas por parte de los/as estudiantes generando una contradicción. Una de las hipótesis que planteamos en este sentido es que quizás estas innovaciones jaquean el modo histórico en que se construyó el oficio de ser estudiante, tal como puede observarse en las voces de los docentes entrevistados:

\begin{abstract}
D: -Ellos están con los jueguitos en red. A veces nos encontramos con este problema, están conectados en red, están en la suya, digamos, uno entra como un espíritu, y por eso uno le tiene reticencia al tic, por eso, porque uno entra, están todos enganchados en un juego, perdidos en la conexión con el que entró o con su entorno y es como que ahí uno muestra un rechazo, pero creo que a ellos les gusta y por ahí estaría bueno empezar a cambiarles un poco el uso a ellos, por ahí hacer como una curvita, empezar a incorporar otras cosas a las computadoras. Como para engancharlos desde otros puntos. (Entrevista 5:15)
\end{abstract}

... los chicos no traen la computadora porque es pesada, porque vienen caminando, en cada curso habrá 405 que la traen y la usan para trabajar en clase, tomar apuntes, anotar fechas de exámenes, copiar en clase. Otros la traen para jugar. (Entrevista 4: 13)

... los chicos me pasan el trapo en... no sé, son buenos usuarios de juegos y ser buenos usuarios de juegos no implica que ellos puedan usar eso para otra cosa. A mí me interesa eso, qué se yo, hay chicos, por ejemplo en la otra escuela, en tercer año yo les pido un trabajo en Word y me dicen "es la primera vez que me lo piden en Word", los chicos no saben usar márgenes, no sé, por decirte algo ¿viste? (Entrevista 9: 29)

También reconocimos la necesidad de que la Institución educativa conforme una comunidad de práctica que acompañe al docente en la integración de TIC a la enseñanza y la consecuente puesta en tensión de los resultados obtenidos. Identificamos otra contradicción relacionada con las demandas sociopolíticas al docente para que integre las TIC a sus propuestas de enseñanza cuando no forman parte de su repertorio de estrategias de enseñanza. Esta contradicción se visualiza en el encuentro entre los/ as docentes y una comunidad que plantea exigencias de integración de TIC pero que lo/a deja solo/a. El acompañamiento está dado por "referentes TIC" integrados a los escenarios escolarespor Programas Nacionales(Conectar Igualdad, por ejemplo). Pero dicho acompañamiento está centrado en los dispositivos y en su funcionamiento, y en muchos casos la relación con los docentes es inexistente:

D: -En mi caso no tengo muchas dificultades, en el sentido técnico me gusta mucho el tema de la informática, lo que sí no tengo yo mucha relación con el TIC de la escuela (referente, cargo) por ejemplo cuál es la clave para ingresar para que los chicos ingresen, yo por ahí hay escuelas que no sé o no conozco al referente TIC, no tengo relación con el tic o sea que... les tengo que preguntar a los profes cuál es la clave para subir un archivo y eso es porque el sistema propio de la escuela no lo conozco mucho, falta relación con el tic, debe ser eso...(Entrevista 3:11)

En el segundo Sistema de Actividad, encontramos estudiantes que hacen uso intensivo de las TIC en su vida cotidiana, creando expectativas respecto de su competencia digital. La contradicción primaria identificada se relaciona con que los/as estudiantes interactúan en redes sociales exhibiendo su mundo privado mientras reciben demandas 
sociales para utilizar sus competencias digitales en la construcción de conocimiento. Se evidencia el desconocimiento de la potencialidad que ofrecerían otros dispositivos digitales como los celulares, que en algunos casos hasta son sancionados por la propia comunidad:

... la tecnología está presente todo el tiempo con los celulares... muchas veces hay que buscarle la vuelta para que esa sea también una herramienta que se pueda utilizar, en general hay una bajada de línea... de que no se puede usar el celular pero yo creo que se pueden ir incorporando y si uno lo prohíbe se produce la clandestinidad... es una herramienta válida y acá sí que hay Internet, y eso hace que uno se replantee las técnicas de evaluación... no preguntar definiciones. Yo no voy a estar juntando los celulares, por un lado es una pérdida de tiempo, y además si pasa algo,no es mi rol... (Entrevista 4: 13)

Las prohibiciones operan a nivel institucional y jurisdiccional, con normativas sancionatorias que han debido ser derogadas. En este sentido, es posible y necesario promover intervenciones que excedan los marcos instrumentales para vincularlas al desarrollo comprensivo de los aprendizajes. La sola utilización del celular para buscar o corroborar información refuerza su uso instrumental y continúa obturando la posibilidad de que el/la docente recupere en parte su autoridad pedagógica en términos epistemológicos. Las prácticas de los/as estudiantes de nivel medio, usuarios de dispositivos digitales y fundamentalmente de redes sociales, configuran una modalidad de atención distribuida que habilita prácticas multitarea, confrontando el tradicional desarrollo de mentes letradas de la escuela. La omnipresencia de dispositivos digitales personales, con sus pantallas individuales y conectividad, redefinen la estructura materialdel aula y la estructura comunicacional de la clase con su propuesta de enseñanza frontal, simultánea y homogénea (Dussel, 2010). Laslimitaciones del tiempo y el espacio se desdibujan cuando los/as estudiantes, mediante sus celulares, corroboran en línea las explicaciones, capturan imágenes del pizarrón, graban videos que comparten en las redes sociales con notorias repercusiones; la escuela continúa fuera de hora en blogs o páginas de Facebook de algunas materias. Esta particular configuración subjetiva desafía la linealidad secuencial y las reglas de comprensión y producción textual como resultado del cúmulo de experiencias mediadas por dispositivos digitales, modificando su manera de aprender, procesar información y resolver problemas.

El acceso al conocimiento está mediado por dispositivos mediante una red extensa de links y un lenguaje global y flexible alineado a códigos audiovisuales masivos (imagen, sonido, animación, texto, etc.). Un mundo fragmentado y veloz posibilita rasgos cognitivos novedosos donde los/as estudiantes construyen saber con los recursos disponibles, organizan tareas con criterios personales y reutilizan producciones propias o ajenas, desafiando las nociones clásicas de autoría y propiedad intelectual.

Por ello, ante el repudiado "corte y pegue" es importante revisar, por ejemplo, las consignas de escritura de textos que existen en Internetapelando a la validación y reformulación de la información:

D: -A mí no me enoja que saquen una foto de un texto, vos tenés que darles otra cosa que antes no dabas, no podes repetir como un loro. Vos tenés que ayudarlos a asociar conocimiento, a construir conocimiento. No más esta clase que tenía yo, con la hojita recitando como un loro no existe, vos tenés que, o sea, vos lo que tenés que hacer es guiar lo que encuentran, suponete en una monografía te pasa que vos les das, yo doy consignas muy sesgadas en monografías, suponete para evitar también esto, porque si no te vienen, bueno, hagan una monografía sobre la adolescencia, te vienen 15.000 monografías de cualquier lado ¿sí? Hay que hacer, me parece que hay que trabajar un poco más, pero no podés resistir algo que ya está. En realidad lo que hay que hacer es 
capacitarse uno, tratar de dar otra clase que vaya más allá de lo que puedan encontrar en cualquier lado. No es fácil, a veces vos venís con algo y el chico si vos le diste... si en la primera clase vos le explicaste que ibas a usar el realismo mágico, el chico al otro día sigue inquieto y se te va a Wikipedia y te sabe todo de realismo mágico, vos tenés que tratar de ir un poco más allá. Sí es un desafío, sí es un desafío, en algunos cursos de chicos muy tecnológicos, los pibes te superan. No me han pasado cosas traumáticas, realmente te digo. Vos tenes que ir más allá de... tenés que pensar una clase que les dé otra cosa que no encuentren en Wikipedia. (Entrevista 2:9)

Reconocer experticia tecnológica en nuestros/as estudiantes, aunque sea centrada en una sola dimensión de la CD, acarrea contradicciones en la división del trabajo y en las reglas. En este sentido, el/a estudiante porta un saber tecnológico instrumental ligado a particulares lógicas de apropiación por fuera del ámbito escolar y, en muchos casos, el/a docente carece de esos saberes, invirtiendo así los roles construidos históricamente. La doble experticia permitía al docente sostener su poder y autoridad. Sin embargo, al requerírsele saberes tecnológicos, hay una parcela a negociar con estudiantes que suelen ser más expertos en la dimensión instrumental.

D: -En realidad ellos saben usar todo y ellos te aportan como unos know how que están buenos, porque son... refrescan tu práctica, por decirlo en algún punto. En realidad hay millones de cosas que vos podés hacer. Yo armé una nube virtual en Facebook, ahí subo los programas, ellos me hacen preguntas que si mi celular anda bien, cosa que yo me tengo que comprar uno mejor ¿no?, yo recibo las inquietudes, contesto y ellos son nativos digitales, ellos tienen mucho más know how que yo, entonces eso como que a mí me, digamos, a ellos les sirve para hacer una mejor cursada, a mí me sirve para mejorar contacto con ellos, a mí me parece bueno, sí. (Entrevista 2:7)

Derivado de estos dos Sistemas de Actividad, proponemos la emergencia de un tercer Sistema de Actividad, que consideramos potente para la construcción del diálogo entre saberes. Su emergencia está dada por la contradicción primaria que supone el ingreso de nuevas subjetividades conformadas en los actuales entornostecnoculturales (SITEAL, 2014) de la Convergencia Digital (Canclini, 2007), que tensionan las interacciones entre los componentes de este Sistema construido en la modernidad:

E: -¿Sabes qué tipo de redes sociales utilizan tus estudiantes?

D: Las que me cuentan ellos, mucho todas tienen, tienen Instagram, Youtube, la de los vídeos, Twitter, con algunos interactúo en Twitter. Facebook, el Facebook es como algo bastante familiar, por ejemplo de la nube virtual yo tengo contacto con los que me preguntan todo el tiempo[...]. Instagram por ejemplo yo no lo he usado, no tengo idea cómo es. Para lo que yo hago, la que más utilizan -que ellos me mandan por ejemplo el avance de las tareas, y yo se los devuelvo y así- es Facebook. Incluso por ejemplo a mí me ha pasado de algunos que no vienen más y tengo que escribir algo, los buscás en Facebook y están y les escribís, o chicos que dejan el colegio, y le preguntás: “mirá ¿qué pasó?” (Entrevista 2:9)

A partir de su inmersión cotidiana y permanente,las subjetividades de nuestras infancias y juventudes se configuran en términos de prótesis electrónicas(SITEAL, 2014). Relaciones y valoraciones caracterizadas por la velocidad, la aceleración, la deslocalización, la desregulación del tiempo (instantaneidad) y la indiferenciación entre lo privado y lo público en el mundo virtual y el mundo real.

En este sentido, analizamos prácticas de enseñanza mediadas por TIC disruptivas con relación a las rutinas de la cultura escolar tradicional. Captan el interés y la atención, ayudando a los/as estudiantes a poner en juego procesos de comprensión y favoreciendo 
que los mismos den sentido a su experiencia escolar. Así, por ejemplo, identificamos la elaboración de una WIKI que permitió no solo vivenciar los cambios superficiales que introducen las nuevas tecnologías en las prácticas de lectura y escritura, sino una comprensión de la escritura como un acto epistémico en el que el sujeto se transforma y no solo da cuenta de algo que ya sabe. En este caso, nos preguntamos ¿qué ayudas podemos ofrecer a nuestros/as estudiantes para lograr nuevas versiones producidas a partir de la escritura colaborativa mediante aplicaciones digitales sincrónicas y asincrónicas? Además de afrontar las condiciones materiales en que se realiza esta innovación (soledad, carencia de recursos e infraestructura, entre otros) ¿ cómo afrontar las propias resistencias de nuestros/as estudiantes a innovaciones que les requieren desarrollar otros saberes y habilidades para una práctica que han naturalizado?

Las contradicciones descriptas obligan a reformular el contrato pedagógico otorgando mayor autonomía al estudiante en términos de ejecución de tareas y requiriendo al docente que conquiste su autoridad pedagógica. Esta ya no sería inherente al proceso de enseñar y aprender, sino que debe construirla en términos epistemológicos para desarrollar las otras dimensiones de la competencia digital. Construir Saberes Docentes Tecnológicos como herramientas de mediación permitiría desarrollar competencias digitales para convertir información en conocimiento, interactuar y colaborar en comunidades, crear y/o reelaborar contenidos digitales, navegar de manera segura e imaginar nuevos usos de la tecnología.

Las prácticas de enseñanza analizadas se desarrollan en un escenario atravesado por la omnipresencia de las pantallas, los medios masivos de comunicación y tecnologías digitales capaces de modelar las prácticas sociales y transformar los procesos de producción, intercambio y negociación de significados.Ofrece un contexto de aprendizaje por fuera de la escuela, signado por la vivencia, la fascinación y la sobreabundancia de información. Si bien estimula una nueva modalidad atencional distribuida, fundamentalmente, manifiesta demandas sociopolítico-laborales de jóvenes multitarea, hiperconectados, capaces de co-crear, realizar búsquedas de información mediante filtrados rápidos y tomar decisiones eficientes mientras interactúan de manera multilineal, en paralelo y en redes.

\section{Conclusiones}

A partir de losanálisisrealizadosenlapresente investigación,pudimosreconocerelmod oenque las TIC median en las prácticas de la enseñanza. Una característica de estos escenarios es la generalización del uso de las TIC; tanto docentes como estudiantes las han incorporado en sus actividades escolares. Aparecen ciertas diferencias con tiempos pasados en los que claramente las resistencias docentes a las incorporaciones de las TIC eran mayores.Tal como hemos señalado, el escenario condiciona, y en muchas oportunidades limita, las posibilidades de uso y de innovación con las TIC; sin embargo, desde la Teoría de la Actividad el escenario no es un simple telón de fondo, sino que permite la generación de diversos ambientes caracterizados por las relaciones que se van estableciendo entre los distintos componentes del Sistema de Enseñanza (Engeström, 2001b; Lave, 1991; Sepúlveda, 2005).

A partir del análisis del trabajo de campo y, en particular, de la $1^{a}$ Dimensión:condiciones del escenario, con sus respectivos indicadores: Tipos de TIC y Materialidad, pudimos reconocer cómo los Programas Nacionales de incorporación de tecnologías a las escuelas (como netbooks, aulas digitales, etc.) favorecieron el uso de las TIC en las aulas. Gran parte de los/as docentes entrevistados/as dieron cuenta de una valoración positiva de las TIC y no se visualizaron resistencias a su incorporación; por el contrario, 
manifestaron su intención de incluirlas en las propuestas de enseñanza y capacitarse al respecto, reconociendo el atractivo que representan para los/as estudiantes.

$\mathrm{Al}$ analizar la relación de los/as docentes con las TIC, observamos que la mayor parte de ellos/as se encuentra transitando lo que se denomina niveles de adopción y/o de adaptación de la tecnología, realizando un uso predominantemente instructivo de las mismas (Christensen y Knezek, 2001). Si bien muchas de estas actividades se orientan a mejorar aquello que se viene haciendo, también se observan algunas experiencias novedosas con las TIC.

Cada vez más comienzan a generarse estrategias que implican niveles complejos de apropiación de las TIC en las propuestas de enseñanza, favoreciendo futuras instancias de innovación en las que podría usarse la tecnología de maneras inéditas. Podríamos decir, entonces, que no solo los/as estudiantes han ido cambiando, sino también los/ as docentes. Creemos que parte de estos cambios se deben a que actualmente muchos/ as de los/as docentes se encuentran en capacitación sobre usos de las TIC o ya han incursionado en algún tipo de formación virtual. Sin embargo, parecería que todavía esto depende del esfuerzo individual del docente y no se vislumbran acciones globales que permitan generalizar el uso de las herramientas tecnológicas en las instituciones educativas.

Es de destacar que en los currículum de educación obligatoriaanalizados, el desarrollo de Competencias Digitales no aparece como un contenido y tampoco en los de Formación Docente. Por cuanto no nos tiene que sorprender que los/as docentes no se sientan preparados para articularlos en sus propuestas de enseñanza. Sumado a esto, las capacitaciones relevadas en el estudio, en su gran mayoría,ofrecen un enfoque único sobre la integración de la tecnología a la enseñanza,mientras que los/as docentes desarrollan su tarea en contextos de enseñanza y aprendizaje cada vez más diversos y,por lo mismo, complejos.

Aun en escenarios desfavorables como en los que suelen desempañarse los/as docentes, pueden generarse ambientes que propicien prácticas enriquecidas con el uso de la tecnología. Esto tendrá mayores posibilidades de concretarse a medida que se generen los espacios donde el/la docente pueda reflexionar sobre todo lo que concierne a la complejidad del trabajo con las TIC, así como también políticas de formación que acompañen dichas reflexiones parapasar de la fascinación por la herramienta a la fascinación por la pedagogía.

En algunos casos,se trata de una incorporación fuera de planificación, es decir que no se hace un uso pedagógico programado de las mismas, sino que es un elemento incorporado para hacer más agradable la clase, para captar la atención de sujetos de aprendizaje mediatizados, reduciéndolo a una prótesis mediacional. En estas condiciones, los/as docentes han desarrollado una representación de las TIC como prótesis del proceso de enseñanza y aprendizaje. Con esto nos referimos a que se las considera una herramienta para captar la atención de los/as estudiantes, para hacer más llamativo el conocimiento estructurado y transmitido de manera tradicional, pero con nuevos atractivos (videos, imágenes, gráficos, etc.).

Los/as docentes consideran la institución como una suerte de escenario que condiciona su propia práctica. En este sentido, lasnetbooks distribuidas por el plan Conectar Igualdad son la principal, y en muchos casos la única, herramienta tecnológica disponible en las voces relevadas. Cabe señalar que al llevar a cabo esta indagación, la mayoría de los/as estudiantes poseían teléfonos celulares inteligentes, sin embargo, no se los consideraba una herramienta a ser recuperada en términos pedagógicos. Las condiciones materiales estarían obturando, por lo tanto, la posibilidad de uso de las 
TIC; un gran porcentaje de docentes manifestaron que parte de las dificultades para incorporarlas en sus propuestas se debían a estas condiciones materiales básicas no garantizadas (Internet e Intranet casi inexistentes en las instituciones).

Por otro lado, y a nivel de las Direcciones de los establecimientos analizados, no identificamos proyectos educativos que acompañen las propuestas docentes; por el contrario, en los dichos de los/as docentes reconocimos que la incorporación de las TIC a sus propuestas de enseñanza se vinculan con intencionalidades individuales.

Finalmente, insistimos en que el ingreso de nuevas subjetividades tecnoculturales está generando contradicciones en el Sistema de Enseñanza, que insiste en abordarlas con los instrumentos materiales y simbólicos construidos históricamente. Aquí coincidimos con Engeström (2001b) en que estas contradicciones no pueden ser solucionadas con acciones individuales y probablemente se agraven con el tiempo, debilitando aún más la ya desjerarquizada autoridad pedagógica.

El diálogo entre saberes entre la cultura escolar y la cultura digital en el Nivel Mediorequiere comunidades de práctica que promuevan la formulación y reflexión sobre nuevos repertorios de actuación para hacer usos más complejos y significativos de las tecnologías digitales y posibiliten el desarrollo de competencias digitales docentes. Planteado así el ambiente, necesariamente el escenario también se vería perturbado, en tanto supone mayor democratización de la escuela en relación con las interacciones que se gestan, con los modos de acceso al conocimiento y, en definitiva,con su construcción, resignificando la mirada histórica relacionada con el disciplinamiento y la reproducción. 


\section{Bibliografía}

" Baquero, R. y Terigi, F. (1996). En búsqueda de una unidad de análisis del aprendizaje escolar.Apuntes pedagógicos(2), Buenos Aires,UBA.

" Bauman, Z. (2013). La cultura en el mundo de la modernidad líquida. Buenos Aires: FCE.

»Canclini, N. (2007). Lectores, espectadores e internautas. Barcelona: Gedisa.

" Christensen, R. y Knezek, G. (2001). Las etapas de adopción como medida de integración de la tecnología. En Morales, C., Ávila, P.; Knezek, G. y Christensen, R. (Eds.).El punto de vista de los usuarios de las nuevas tecnologías en educación: estudio de diversos países. México: ILCE.

》 Cole, M. (1999). Psicología Cultural. Madrid: Morata.

"Daniels, H. (2003). Vygotsky y la pedagogía. Barcelona: Paidós.

"Díaz Barriga, F. (2003). Cognición situada y estrategias para el aprendizaje significativo. Revista Electrónica de Investigación Educativa, 5 (2).Recuperado de: $<$ http://redie.ens.uabc.mx/volsnoz/contenido-arceo.html> [consulta: 17/og/2012].

»Dussel, I. (2010). Aprender y enseñar en la cultura digital. VI Foro Latinoamericano de Educación. Educación y nuevas tecnologías: los desafíos pedagógicos ante el mundo digital. Buenos Aires: Santillana.

» Edwards, V. (1985). Los sujetos y la construcción social del conocimiento.Chile: DIE.

»Engeström, Y. (2001a). El aprendizaje expansivo en el trabajo: hacia una reconceptualización teórica de la actividad. Journal of Education and Work,14 (1). Recuperado de:https://www.academia.edu/26972546/El_aprendizaje_expansivo_ en_el_trabajo_hacia_una_reconceptualizaci\%C3\%B3n_te\%C_\% B3rica_de_la_ actividad [consulta: 17/02/2014].

"Engeström, Y. (2001b). Los estudios evolutivos del trabajo como punto de referencia de la teoría de la actividad: el caso de la práctica médica en la asistencia básica. En Chaiklin,S. y Lave, J. (Comps.).Estudiar las Prácticas. Buenos Aires: Amorrortu.

"IBARÓMETRO (2010). Evaluación del impacto del Programa Conectar Igualdad.

» INTEF (2017). Marco de Competencia Digital Docente. Recuperado de: http:// educalab.es/documents/10180/12809/MarcoComunCompeDigiDoceV2. pdf[consulta: 03/03/2018].

" Koehler, M. J.; Mishra, P. y Cain, W. (2015).¿Qué son los Saberes Tecnológicos y Pedagógicos del Contenido (TPACK)?Virtualidad, Educación y Ciencia, 10 (6), pp. 9-23. Recuperado de:http://revistas.unc.edu.ar/index.php/vesc/article/view/11552/11983 [consulta: 10/2015].

》 Lave, J. (1991). La cognición en la práctica. Buenos Aires: Paidós.

》 Litwin, E. (2008). El oficio de enseñar: condiciones y contextos. Buenos Aires: Paidós.

》 Lyotard, J. (1991). La posmodernidad. Barcelona: GEDISA.

" Martín Barbero, J. (2001). Tecnicidades, identidades, alteridades: des-ubicaciones y opacidades de la comunicación en el nuevo siglo.Diálogos de la Comunicación(64). México: FELAFACS.

» Matozo Martínez, V. (2016). La inclusión digital desde Conectar Igualdad. 
(Ponencia). $9^{\circ}$ Jornadas de Sociología de la Universidad Nacional de La Plata.

»Salomon, G. (1993). Cogniciones distribuidas. BuenosAires: Amorrortu.

»Sepulveda, G. (2005). Esbozo de la Teoría de la Actividad. Universidad Frontera. Chile.Recuperado de:www.innovat.cl[consulta: 10/06/13].

» SITEAL (2014). Informe sobre tendencias sociales y educativas en América Latina. Políticas TIC en los sistemas educativos de América Latina. Recuperado de: http:// www.tic.siteal.org/sites/default/files/stic_publicacion_files/siteal_informe_2014_ politicas_tic.pdf [consulta: 20/04/14].

»Vattimo, G. (1990). Posmoderno: ¿una sociedad transparente? Barcelona: Paidós.

"Vygotski, L. (2000). El desarrollo de los procesos Psicológicos Superiores. Barcelona: Crítica.

»Wenger, E. (2001). Comunidades de práctica, aprendizaje, significado e identidad. Barcelona: Paidós.

»Wertsch, J. (1993). Voces de la mente. Madrid: Visor.

\section{Rita De Pascuale}

Especialista y Magister en Didáctica, Universidad de Buenos Aires. Profesora en Ciencias de la Educación, Instituto de Formación Docente $\mathrm{N}^{\circ} 3$, Bahía Blanca. Directora del Centro de Estudios Didácticos del Comahue. Profesora e Investigadora, Universidad Nacional del Comahue. ritapascuale@gmail.com

\section{Sonia Sansot}

Magister en Psicología Cognitiva y Aprendizaje, Facultad Latinoamericana de Ciencias Sociales (Argentina). Profesora en Ciencias de la Educación, Universidad Nacional de La Pampa.Profesora e Investigadora, Facultad de Ciencias de la Educación, Universidad Nacional del Comahue. soniasansot@gmail.com 